\title{
Reconstruction after Subtotal Sacrectomy for Sacral Ewing's Sarcoma Using Tibial Allograft Strut Grafting: A Case Report
}

\author{
Ryosuke Hirota Makoto Emori Yoshinori Terashima Kousuke Iba \\ Noriyuki lesato Ryunosuke Fukushi Mitsunori Yoshimoto \\ Toshihiko Yamashita \\ Department of Orthopaedic Surgery, Sapporo Medical University School of Medicine, \\ Sapporo, Japan
}

\author{
Keywords \\ Ewing's sarcoma $\cdot$ Subtotal sacrectomy $\cdot$ Tibial allograft $\cdot$ Reconstruction
}

\begin{abstract}
We present the case of a 15-year-old girl. Two months after becoming aware of pain, she was diagnosed with a sacral tumor and referred to our department. She was diagnosed with a sacral Ewing's sarcoma; after chemotherapy, it was determined that the tumor could be resected, so surgical treatment was performed. The sacrum and ilium were partially resected at the lower end of $\mathrm{S} 1$, and the lumbar vertebrae and pelvis were fixed with a pedicle screw and two iliac screws on each side of $L 3$, and the sacral resection was reconstructed with a tibial strut allograft. No tumor recurrence or metastasis has been observed 1 year postoperatively. She developed bladder and rectal dysfunction, but she remained independent in activities of daily living and her daily life was not limited. The bone fusion in the reconstructed area confirmed the lack of instrumentation looseness. Surgical treatment for sacral Ewing's sarcoma was performed to cure the patient. We believe that the tibial allograft contributed to the patient's ability to walk on her own due to its high mechanical stability. Postoperative bone healing was observed with the same material, suggesting that the tibial allograft is useful for similar procedures.

\section{Introduction}

Ewing's sarcoma (ES) is a small round cell neuroectodermal neoplasm. It is the second most common primary bone tumor in children and young adults. A multidisciplinary treatment approach is needed for this highly malignant tumor. ES of the pelvis has a particu- 

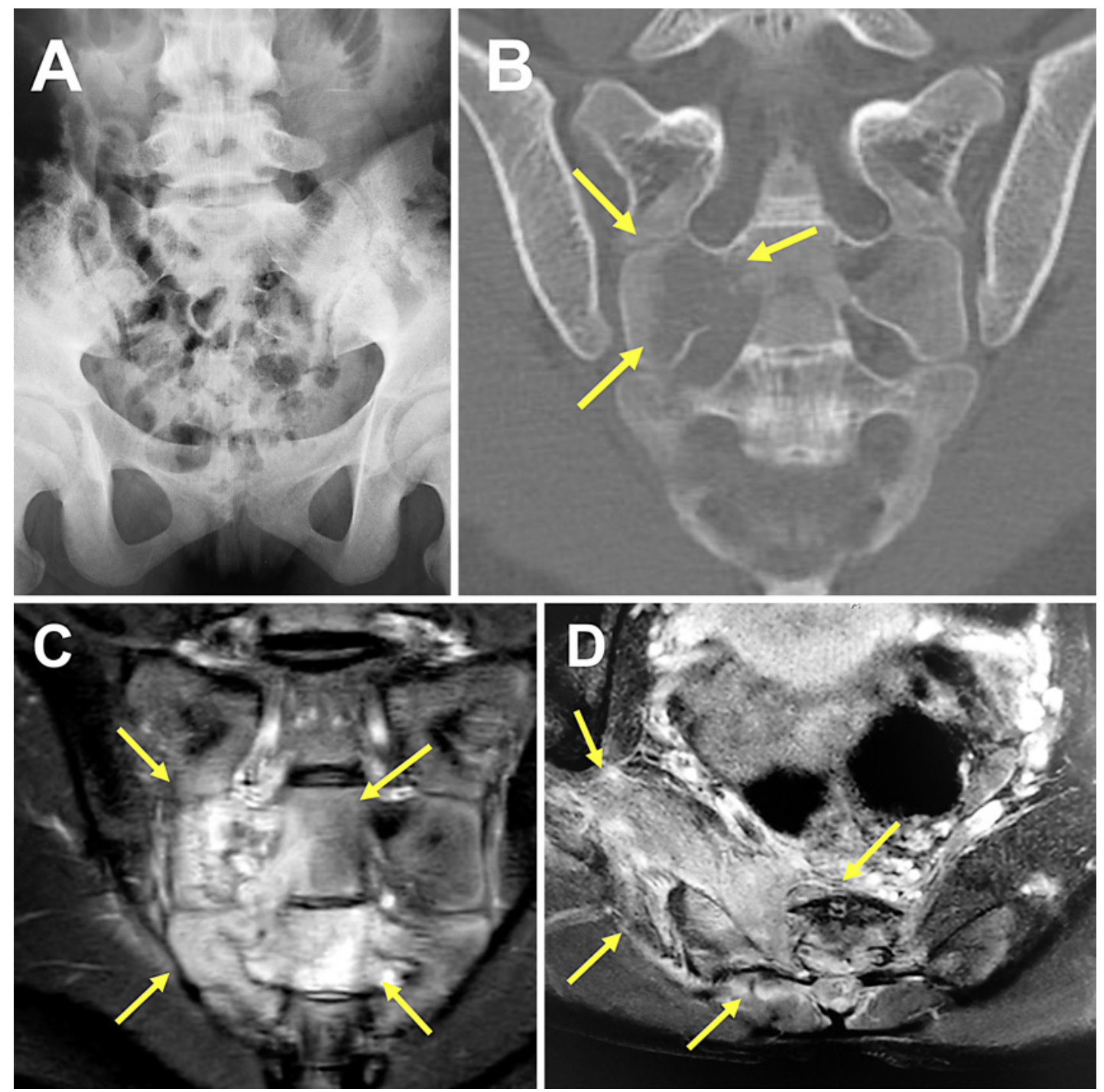

Fig. 1. Radiograph of the pelvis (A), computed tomography in the coronal plane (B), and magnetic resonance imaging in the coronal plane (C) and in the axial plane (D). A destructive lesion on the right side of the S2 sacrum with no calcification was observed (yellow arrows).

larly poor life prognosis. Surgical treatment for ES is considered to provide a better prognosis than radiotherapy if a safe resection margin can be secured. However, in cases of surgical difficulty, radiotherapy or proton beam therapy is generally considered. We report on a case of sacral ES with good postoperative local control after extensive resection for radical cure.

\section{Case Report}

A 15-year-old girl presented with right-buttock pain for 2 months. She had no history of trauma, and her medical history was unremarkable. On physical examination at our hospital, she had pain on movement in her right hip. However, there was no swelling, redness, or 

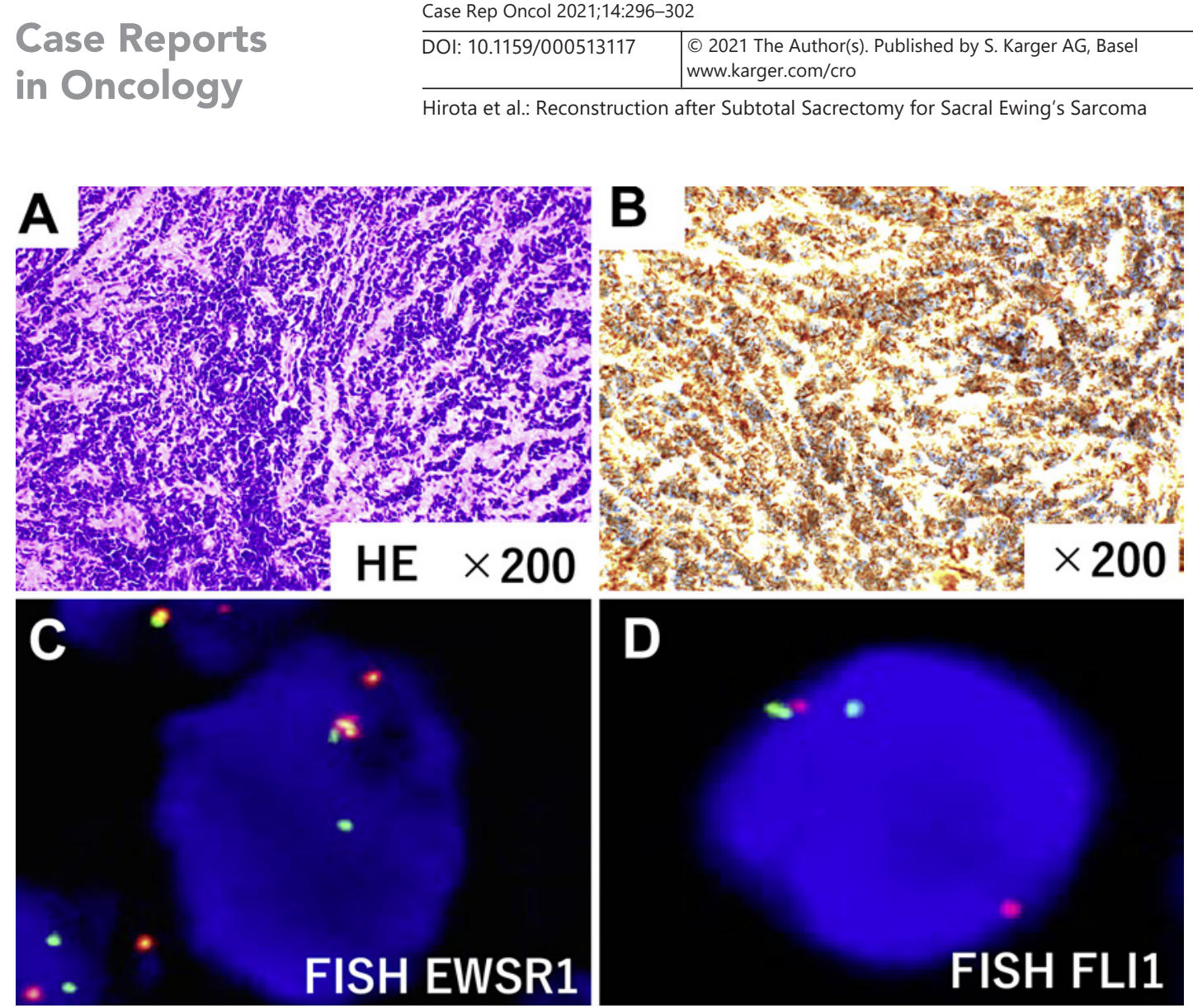

Fig. 2. Histopathological examination (A) and immunohistochemical analysis (B). Fluorescence in situ hybridization (C, D) showed an ESWR1 split signal in 54\% and an FLI1 split signal in 56\%.

burning sensation in the same area. There were no significant neurological findings, such as numbness or muscle weakness in the lower extremities.

Blood investigations revealed a white blood cell count and C-reactive protein level of $4.9 \times 10^{9}$ cells/L (neutrophils, 59.0\%; lymphocytes, 36.0\%; monocytes, $4.0 \%$ ) and $1.69 \mathrm{mg} / \mathrm{dL}$ (normal: $<0.30$ ), respectively. On radiological investigations, her X-ray scans showed no obvious bone destruction (Fig.1A); however, a destructive lesion on the right side of the S2 sacrum with no calcification was observed on computed tomography (CT) (Fig. 1B). On magnetic resonance imaging, the lesion measured $63 \times 30 \times 11 \mathrm{~mm}$; it was isointense on T1-weighted imaging and showed mixed low-high signal intensity on short TI inversion recovery-weighted imaging. The signal changes expanded beyond the mid-sacral region and into the spinal canal (Fig. 1C, D). The results of a chest CT scan and positron emission tomography-CT performed to rule out metastatic disease were negative. Based on these findings, we suspected that the lesion was an osteomyelitis or ES. An open incisional biopsy was performed. Histologically, the tumor was composed of circular cells that were proliferating in a cord-like, part-sheet-like structure, and were necrotic (Fig. 2A). Immunohistochemical analysis revealed that the tumor was positive for CD99 (Fig. 2B). A diagnosis of ES was made. This was supported by fluorescence in situ hybridization, which showed an ESWR1 split signal in 54\% and an FLI1 split signal in 56\% (Fig. 2C, D).

The patient was treated with neoadjuvant chemotherapy using the VDC/IE regimen comprising vincristine, doxorubicin, and cyclophosphamide alternating with cycles of ifosfamide and etoposide. The chemotherapy caused 73\% tumor shrinkage (Fig. 3A, B). We performed wide excision and reconstruction. 


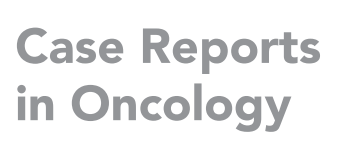

\begin{tabular}{l|l}
\hline Case Rep Oncol 2021;14:296-302 \\
\hline DOI: 10.1159/000513117 & $\begin{array}{l}\text { @ 2021 The Author(s). Published by S. Karger AG, Basel } \\
\text { www.karger.com/cro }\end{array}$ \\
\hline
\end{tabular}

Hirota et al.: Reconstruction after Subtotal Sacrectomy for Sacral Ewing's Sarcoma

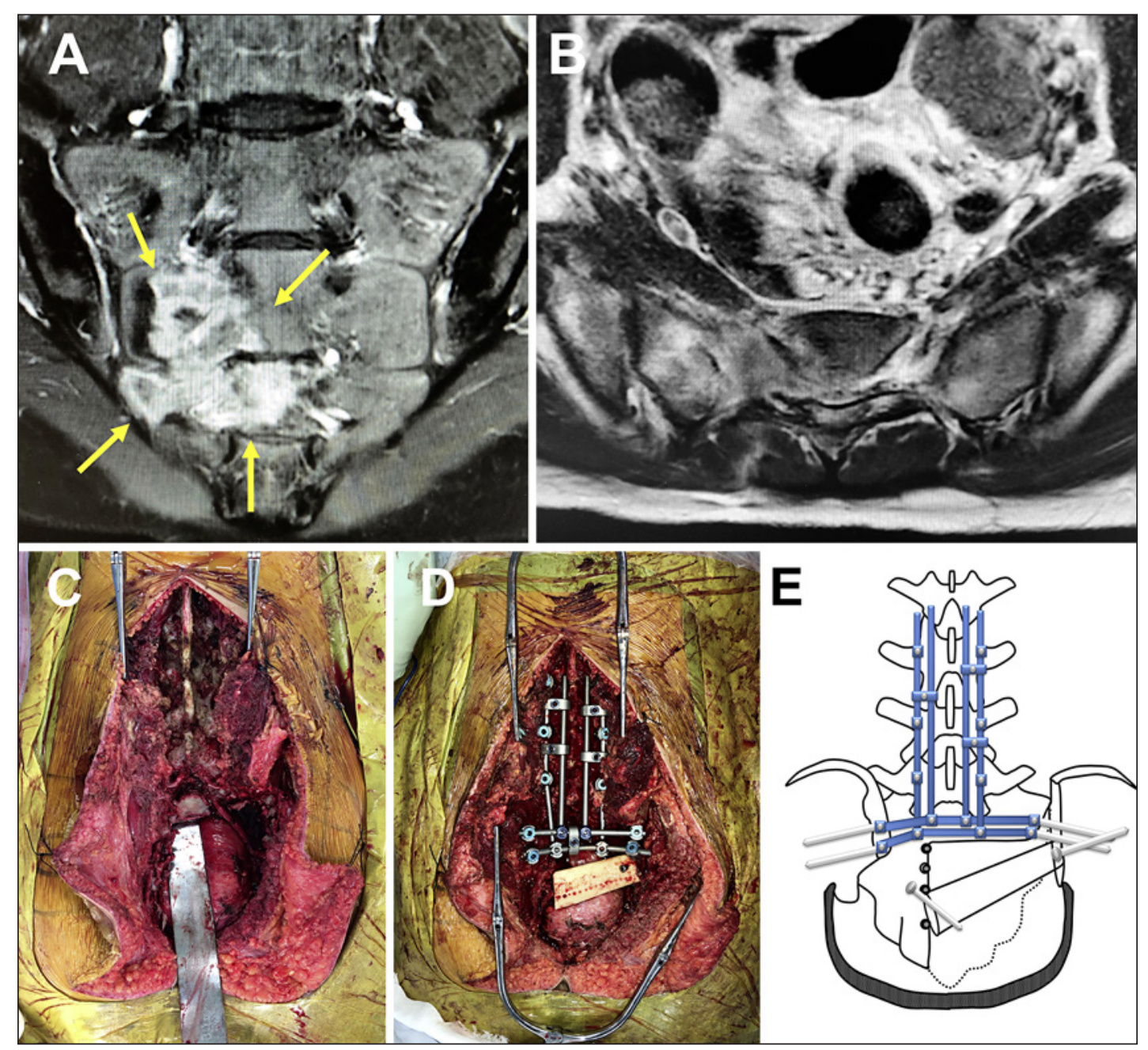

Fig. 3. Computed tomography (A) and magnetic resonance imaging of the pelvis (B) after neoadjuvant chemotherapy. Neoadjuvant chemotherapy caused 73\% tumor shrinkage. We performed extensive pelvic resection and reconstruction using tibial allograft strut grafting with double posterior rod instrumentation (C-E).

\section{Surgical Procedure}

First, the patient was placed in the supine position and administered general anesthesia. The incision that was taken in the supine position. We ligated the anterior sacral artery and sacral venous plexus in front of the tumor to reduce intraoperative bleeding. Subsequently, the patient was re-placed in the prone position. A posterior midline incision was made extending from L2 to S4. For the partial sacral resection, the sacrum was resected at the level of the S1 sacral foramen (Fig. 3C). The right sacroiliac joint was included and the left side was resected in the form of a connecting sacral foramen. The cauda equina was severed at the S2 level with the dural canal ligated at the cranial side. The sacral resection was then reconstructed using an allogeneic tibial strut bone graft. L3 to the iliac bone was fixed posteriorly with a dual rod. Moreover, sufficient bone grafting was performed and the allogeneic fibula bone chip was grafted in L3 to the iliac bone (Fig. 3D, E). 


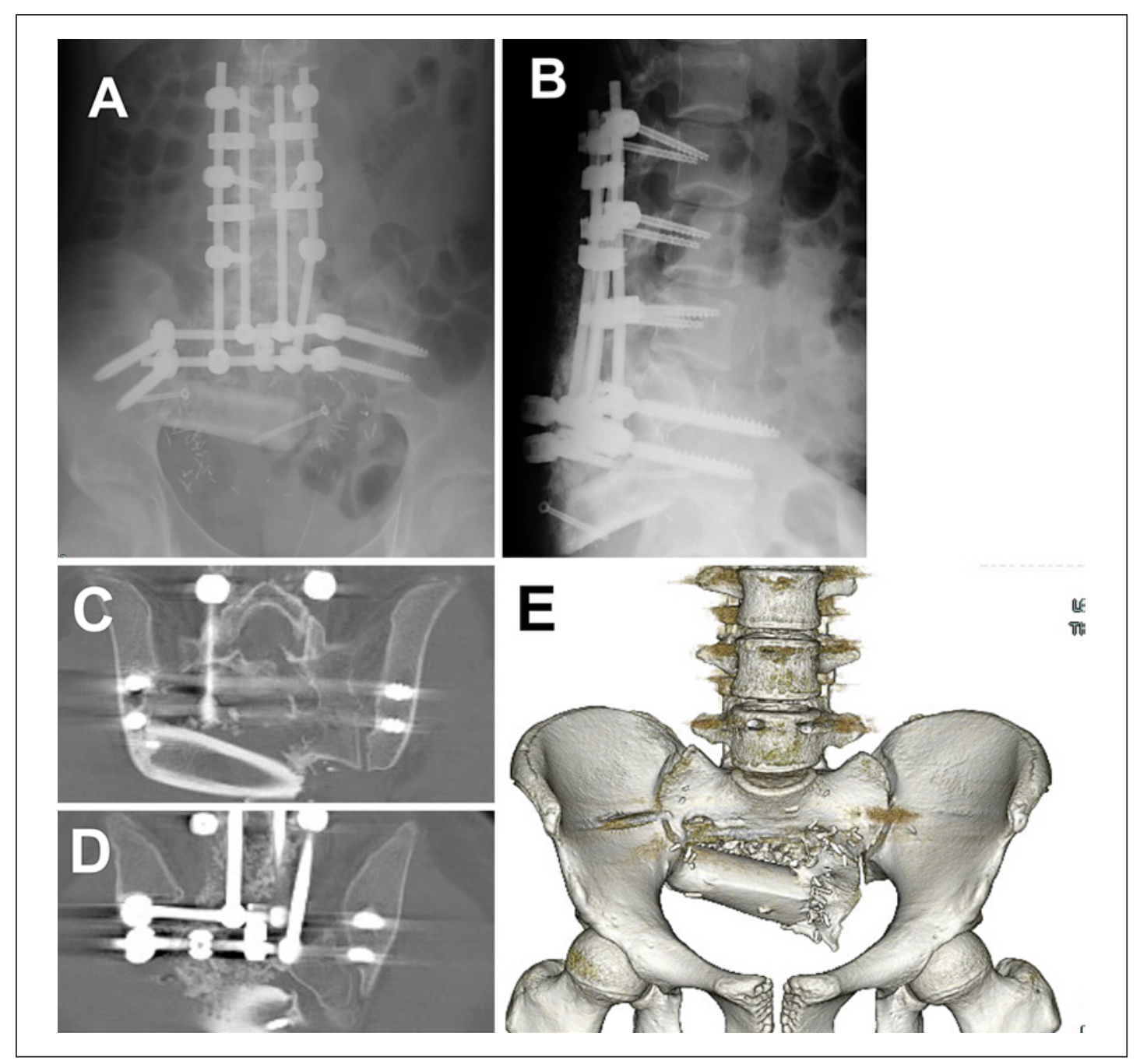

Fig. 4. Postoperative radiographs (A, B) and computed tomography images (C-E) of the pelvis. There was no osteolysis of the reconstructed area and no implant failure.

A blood transfusion was required based on an estimated intraoperative blood loss of $4,900 \mathrm{~mL}$. Histological analysis of the tumor specimens revealed no tumor cells at the tissue margins.

\section{Postoperative Course}

Immediately after surgery, DigniCare (Bard Medical Division, C. R. Bard, Inc., Covington, GA, USA) was used for 2 weeks to drain stool via the rectum to avoid contamination of the wound. Full-weight gait training was started 2 weeks postoperatively, and postoperative chemotherapy was started on day 31 after surgery, when the wound was completely healed. Radiotherapy was not performed, because the tumor was removed with wide margins and there were no viable tumor cells.

At 1 year and 3 months postoperatively, the patient was free of disease (Fig. 4). There was no osteolysis of the reconstructed area and no implant failure. The patient was pain free; she had resumed exercising and could independently perform her daily activities without a 
cane. However, she has a bladder-rectal disorder and is self-catheterizing. Her Musculoskeletal Society Tumor score (MSTS) was 30 and the Toronto Extremity Salvage Score (TESS) was $99.2(119 / 120)$.

\section{Discussion}

ES is the most common malignant bone tumor among children and adolescents, and it requires a multidisciplinary treatment approach [1]. Multimodal therapy, including surgery, radiotherapy, and chemotherapy, has improved the treatment for ES over the past 40 years. The pelvis is the most common site for ES, and tumors that arise in the pelvic area are usually associated with poor survival compared with tumors in the extremities [2]. Disease-free survival among patients with ES is reportedly better for tumors localized in the sacrum than for those in the innominate bones, where these tumors are generally larger [3]. However, the overall 5-year survival rate for sacral ES is only 65\% [3].

Recently, surgery has been increasingly preferred to treat pelvic ES [4]. Chen et al. [5] also reported that surgical treatments are associated with better outcomes for pelvic ES. Several previous studies have compared radiotherapy to surgery, and reported surgery to be superior in terms of event-free survival, overall survival, and local control [6, 7]. In this case, we judged the tumor to be resectable because it had shrunk following chemotherapy.

The important point to be noted during surgical treatment for sacral tumors is to preserve the stability of the spine and pelvis while ensuring a safe tumor margin. In our case, a partial resection of the right sacroiliac joint on the side of the tumor was performed to secure a sufficient resection margin. Although bilateral resection of the sacroiliac joint is recommended to reconstruct the defect for stability [8], it is unclear whether reconstruction is required in unilateral resection such as in the present case. In this case, the caudal half of S1 and the unilateral sacroiliac joint had been resected. The defect was reconstructed to avoid instability considering the patient's age and activity level. Moreover, the spine and pelvis were fixed with a dual rod. Although reconstructive methods such as vascularized peroneal grafting have been reported [9], allogeneic tibial grafting was chosen for tissue preservation in this patient, because she was young and hoped to continue exercising, and the removal of her autologous fibula could affect her motor function. It should be acknowledged that, with surgical resection of the tumor, there is a substantial risk of wound infection due to the long duration of the operation and wound contamination by urination and defecation. Deep wound infection has been reported in $50 \%$ of the patients in studies assessing sacrectomy with reconstruction [10]. In our case, fecal contamination of the wound was avoided by using the DigniCare Stool Management System (device A; Bard Medical Division, C. R. Bard, Inc.). We also believe that sufficient bone grafting may have helped to reduce the dead space under the muscle layer.

\section{Conclusion}

As demonstrated in our case, sacral ES can be managed with extensive resection and reconstruction using allogeneic bone for radical cure. Fusion with a dual rod and allogeneic tibial grafting is mechanically stable and contributes to the acquisition of an early selfpowered gait. The bone defect can be reconstructed using allogeneic tibia, and osteolysis can be achieved, as in our case.

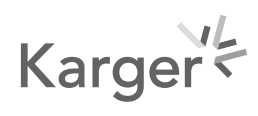




\section{Case Reports in Oncology}

Case Rep Oncol 2021;14:296-302

\begin{tabular}{l|l}
\hline DOI: 10.1159/000513117 & c 2021 The Author(s). Published by S. Karger AG, Basel
\end{tabular} www.karger.com/cro

\section{Statement of Ethics}

Written informed consent was obtained from the patient and her parents for publication of this case report and any accompanying images.

\section{Conflict of Interest Statement}

The authors have no conflicts of interest to declare.

\section{Funding Sources}

No funding was received.

\section{Author Contributions}

R.H. assisted in this operation and wrote the manuscript. M.E. was the attending physician of this patient and performed the chemotherapy. Y.T., N.I., K.I., and R.F. performed the operation. M.Y. and T.Y. edited the manuscript.

\section{References}

1 Fletcher CDM, Bridge JA, Hogendoorn PCW, Mertens F. WHO classification of tumors of soft tissue and bone. WHO classification of Tumours. 4th ed. Lyon: IARC Press; 2013.

2 Mounessi FS, Lehrich P, Haverkamp U, Willich N, Bölling T, Eich HT. Pelvic Ewing sarcomas. Three-dimensional conformal vs. intensity-modulated radiotherapy. Strahlenther Onkol. 2013 Apr;189(4):308-14.

3 Hesla AC, Tsagozis P, Jebsen N, Zaikova O, Bauer H, Brosjö O. Improved prognosis for patients with Ewing sarcoma in the sacrum compared with the innominate bones: the Scandinavian Sarcoma Group Experience. J Bone Joint Surg Am. 2016 Feb;98(3):199-210.

4 Puri A, Gulia A, Jambhekar NA, Laskar S. Results of surgical resection in pelvic Ewing's sarcoma. J Surg Oncol. 2012 Sep;106(4):417-22.

5 Chen L, Long C, Liu J, Xing F, Duan X. Characteristics and prognosis of pelvic Ewing sarcoma: a SEER population-based study. PeerJ. 2019;7:e7710.

6 Ahmed SK, Robinson SI, Arndt CAS, Petersen IA, Haddock MG, Rose PS, et al. Pelvis Ewing sarcoma: local control and survival in the modern era. Pediatr Blood Cancer. 2017 Sep;64(9):e26504.

7 Choi Y, Lim DH, Lee SH, Lyu CJ, Im JH, Lee YH, et al. Role of radiotherapy in the multimodal treatment of Ewing sarcoma family tumors. Cancer Res Treat. 2015 Oct;47(4):904-12.

8 Zhu R, Cheng LM, Yu Y, Zander T, Chen B, Rohlmann A. Comparison of four reconstruction methods after total sacrectomy: a finite element study. Clin Biomech (Bristol, Avon). 2012 Oct;27(8):771-6.

9 Gillis CC, Street JT, Boyd MC, Fisher CG. Pelvic reconstruction after subtotal sacrectomy for sacral chondrosarcoma using cadaveric and vascularized fibula autograft: technical note. J Neurosurg Spine. 2014 Oct;21(4): 623-7.

10 Arkader A, Yang CH, Tolo VT. High long-term local control with sacrectomy for primary high-grade bone sarcoma in children. Clin Orthop Relat Res. 2012 May;470(5):1491-7.

\section{Karger'}

